\title{
LA RADIO UNIVERSITARIA EN COLOMBIA COMO MEDIO DE INTERÉS PÚBLICO
}

\section{COLLEGE RADIO IN COLOMBIA AS A PUBLIC INTEREST MASS MEDIA}

\section{A RÁDIO UNIVERSITÁRIA NA COLÔMBIA COMO MEIO DE INTERESSE PÚBLICO}

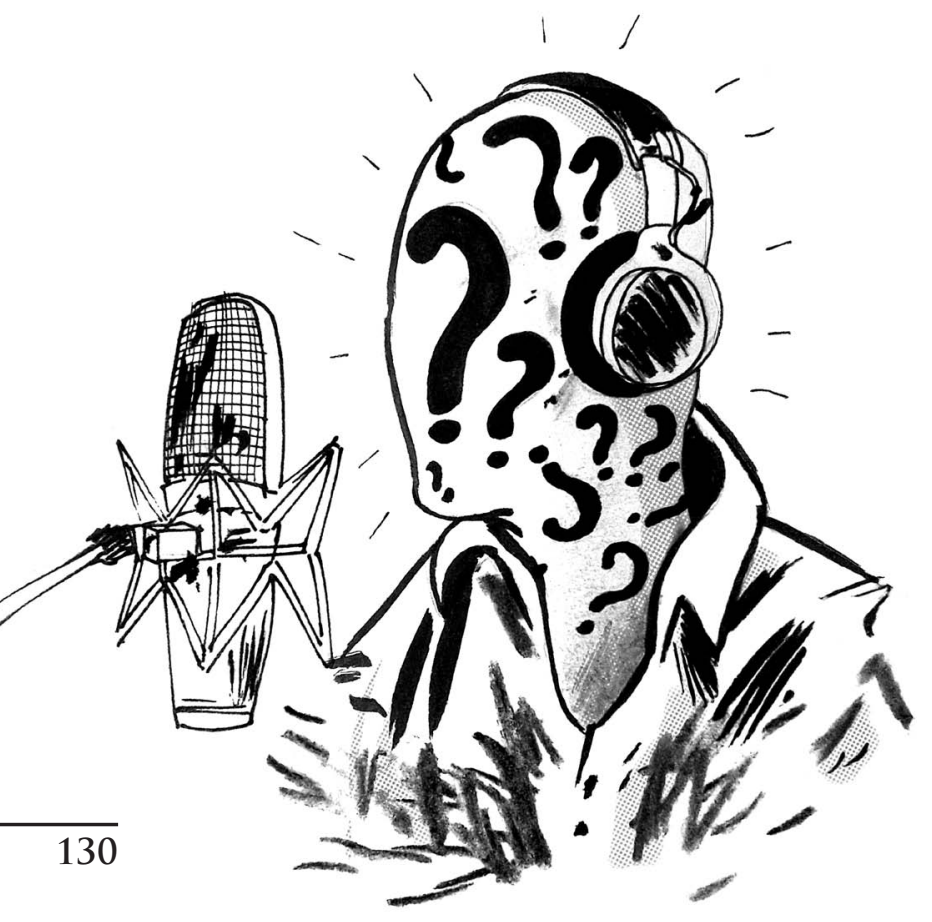

Por:

Ana María Díaz Jordán ${ }^{1}$

Profesora

Universidad Santiago de Cali, Colombia

Universidad del Valle, Colombia

ana.diaz00@usc.edu.co

Resumen: Este artículo realiza una revisión descriptiva de la literatura relacionada con el término 'Radio Universitaria de Interés Público en Colombia', con el objetivo de brindar un contexto sobre su origen y sus características. Según lo dispuesto en la resolución 0415 del 23 de abril de 2010, las radios universitarias, por la orientación de su programación, hacen parte de la radio de interés público, es decir que estas emisoras satisfacen las necesidades de comunicación del Estado con los ciudadanos y comunidades. Pero, de manera independiente a la legislación ¿qué es una radio de interés público? ¿Cuáles son sus principales características? ¿Por qué algunas radios universitarias en el país funcionan con licencias de medios de interés público? ¿Qué literatura se ha escrito sobre el tema? Estos son algunos de los interrogantes que pretende resolver este artículo de revisión ${ }^{2}$.

Palabras clave: radio universitaria, radio de interés público, la radio universitaria en Colombia. 
Abstract: This article provides a descriptive review of the literature related to the term 'Radio University Public Interest in Colombia', with the aim of providing a context about their origin and their characteristics. In accordance with resolution 0415 of April 23, 2010, college radio, by targeting its programming, are part of the radio public interest, these stations meet the communication needs of the state with citizens and communities. But independently of the legislation what is a radio public? What are its main characteristics? Why do some college radio in the country work with media licenses in the public interest? What literature has been written on the subject? These are some of the questions that aims to solve this review article.

Keywords: college radio, radio public interest, the college radio in Colombia.

Resumo: Este artigo faz uma revisão descritiva da literatura relacionada com o termo Rádio universitária de interesse público na Colômbia, com o objetivo de fornecer um contexto sobre a sua origem e as suas caraterísticas. Segundo a resolução 0415 de Abril 23 de 2010, as rádios universitárias, pela sua programação, fazem parte da rádio de interesse público. Em outras palavras, estas emissoras satisfazem as necessidades de comunicação do Estado com os cidadãos e comunidades. Porém, independentemente da legislação, o que é uma rádio de interesse público? Quais suas principais caraterísticas? Por que algumas rádios universitárias no país têm licenças de meios de interesse público? Qual literatura tem sido escrita sobre o tema? Estas são algumas questões que este artigo de reflexão quer resolver.

Palavras-chave: Rádio universitária, rádio de interesse público, a rádio universitária na Colômbia.

\section{Introducción}

En Colombia existe una amplia legislación que regula el servicio de radiodifusión. Entre esas leyes y decretos se puede destacar la Ley 1341 de 2009, que define principios y conceptos sobre la sociedad de la información y la organización de las tecnologías de la información y las comunicaciones, y además crea la Agencia Nacional del Espectro; también el Decreto 4350 de 2009, por el cual se establece el régimen unificado de contraprestaciones por concepto de concesiones, autorizaciones y permisos en materia de servicios de radiodifusión sonora.

Otra norma es la Resolución 0415 de 2010, que hace referencia a la estructura de propiedad de la radio en el país. Sobre ésta última se hará especial énfasis en este documento. Esta resolución aplica para la radio en A.M y en F.M. e indica varias clasificaciones de la radiodifusión sonora: Gestión del servicio, Nivel de cubrimiento, Tecnología de transmisión y Orientación de la programación. 


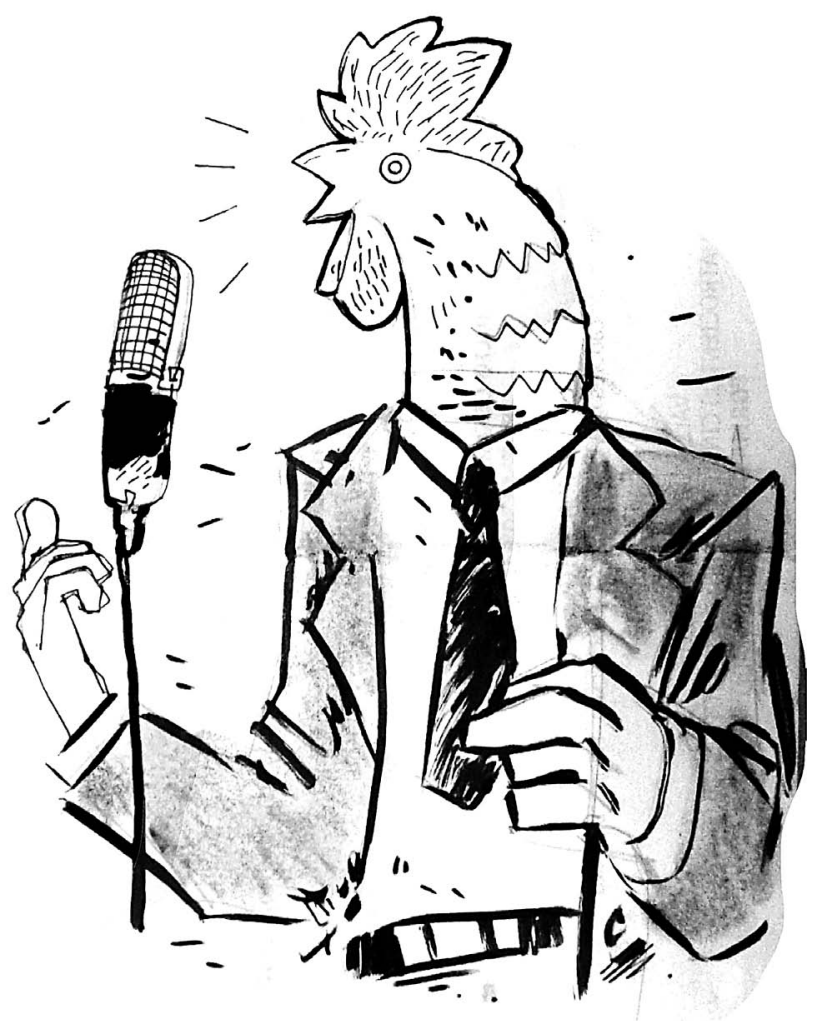

Respecto a la gestión del servicio la radio en Colombia puede ser de gestión directa, la que presta el Estado a través de entidades públicas debidamente autorizadas por la ley o a través de licencia otorgada por el Ministerio de Tecnologías de la Información y las Comunicaciones (MinTic). O de gestión indirecta, que se da mediante contrato o licencia, previa realización de un procedimiento de selección objetiva.

De acuerdo al nivel de cubrimiento la radiodifusión en Colombia se clasifica en estaciones clase $A$ y $B$, destinadas a cubrir áreas más o menos extensas que contienen varios municipios o distritos; estaciones clase $C$ que cubren un solo municipio o distrito y estaciones clase $D$, destinadas a cubrir áreas urbanas y/o rurales o zonas específicas dentro de un municipio o distrito.

Según la tecnología de transmisión, la radio en el país puede ser de Amplitud Modulada (A.M), Frecuencia Modulada (F.M), Radiodifusión digital y nuevas tecnologías.

En función de la orientación de la programación, las emisoras pueden ser: Comerciales, cuando la programación está destinada a la satisfacción de los hábitos y gustos del oyente y el servicio se presta con ánimo de lucro, sin excluir el propósito educativo, recreativo, cultural, científico e informativo. Comunitarias, cuando la programación está orientada a generar espacios de expresión, información, educación, comunicación, promoción cultural, formación, debate y concertación que conducen al encuentro entre las diferentes identidades sociales y expresiones culturales de la comunidad, dentro de un ámbito de integración y solidaridad ciudadana y, en especial, la promoción de la democracia, la participación y los derechos fundamentales de los colombianos que aseguren una convivencia pacífica. De Interés Público, cuando la programación se orienta a satisfacer necesidades de comunicación del Estado con los ciudadanos y comunidades, la defensa de los derechos constitucionales, la protección del patrimonio cultural y natural de la nación, a fin de procurar el bienestar general y el mejoramiento de la calidad de vida de la población. Estas estaciones no tienen ánimo de lucro y no pueden transmitir pauta comercial. Las entidades que soliciten licencias para emisoras de interés público deben asumir su financiamiento técnico, administrativo y de contenido. Las emisoras de interés público se dividen en: 1.Emisoras de la Radio Pública Nacional de Colombia. 2. Emisoras de la Fuerza Pública. 3. Emisoras Territoriales. 4. Emisoras Educativas. 5. Emisoras Educativas Universitarias. 6. Emisoras para atención y prevención de desastres. 


\section{Clasificación del Servicio de Radiodifusión Sonora en Colombia Resolución 0415 de 23 de abril de 2010}

\begin{tabular}{|c|c|c|c|}
\hline $\begin{array}{l}\text { GESTIÓN DEL } \\
\text { SERVICIO }\end{array}$ & $\begin{array}{c}\text { NIVEL DE } \\
\text { CUBRIMIENTO }\end{array}$ & $\begin{array}{l}\text { TECNOLOGÍA DE } \\
\text { TRANSMISIÓN }\end{array}$ & $\begin{array}{l}\text { ORIENTACIÓN DE LA } \\
\text { PROGRAMACIÓN }\end{array}$ \\
\hline $\begin{array}{l}\text {-Gestión Directa } \\
\text {-Gestión Indirecta }\end{array}$ & $\begin{array}{l}\text {-Clase A } \\
\text {-Clase B } \\
\text {-Clase C } \\
\text {-Clase D }\end{array}$ & $\begin{array}{l}\text {-Amplitud Modulada } \\
\text { (A.M). } \\
\text {-Frecuencia Modulada } \\
\text { (F.M). } \\
\text {-Radiodifusión digital y } \\
\text { nuevas tecnologías. }\end{array}$ & $\begin{array}{l}\text {-Comerciales } \\
\text {-Comunitarias } \\
\text {-Interés Público }\end{array}$ \\
\hline
\end{tabular}

Tabla 1.

La Radio de interés público

Más allá de la legislación, es necesario ahondar en el porqué de la concepción de las radios universitarias como radios de interés público; es decir, radios que satisfacen las necesidades de comunicación del Estado con los ciudadanos y las comunidades.

La radio de interés público se materializa a través de diferentes modelos en todo el mundo. Es posible encontrar sistemas de medios denominados de interés público patrocinados por el Estado; también están presentes los casos en que estos medios son financiados a través del canon que pagan los ciudadanos y, en otras situaciones, estos medios deben competir por la publicidad comercial. En cuanto a la orientación de contenidos no existe un modelo consensuado para este tipo de emisoras: algunos países orientan la programación de servicio público a la educación, información y entretenimiento, otros defienden posturas políticas, otros tratan de imitar la programación de canales comerciales o reproducir los contenidos de medios locales y otros buscan la difusión de una identidad de nación (Atkinson y Raboy, 1998).

El servicio público de radiotelevisión en los Estados Unidos (PBS), se creó para cumplir una función educativa y poco a poco se le fue añadiendo la tarea de informar y entretener. Los lineamientos legislativos le asignan a este sistema el objetivo de garantizar la expresión de las minorías excluidas por la dinámica mercantil. En la práctica, existe una gran diferencia respecto a la significación e importancia que se le da a este sistema, en comparación con otros países que tratan de atender, representar y agrupar la nación a través de los medios de interés público. A diferencia de redes comerciales como NBC, CBS y ABC, la red de radiotelevisión pública en Estados Unidos llega a más hogares, pero su índice de audiencia es aproximadamente del 2\% (Atkinson y Raboy, 1998). La principal explicación a esta situación es que el contenido de estos medios generaliza "un gusto limitado y algo clasista a una población que (...) 
no lo acepta y al mismo tiempo, deja la difusión de la cultura popular en manos de organismos a los que podrían importarles menos los conceptos de calidad" (Atkinson y Raboy, 1998, 118).

En Europa también se han creado sistemas de radiotelevisión pública como BBC en Gran Bretaña, RFI en Francia, RAI en Italia y RN en Holanda (Berlin, 2000). En la radio pública de Gran Bretaña pueden distinguirse tres grandes periodos con características específicas: el primero de índole nacional, el segundo de carácter popular y el último pluralista, que trabaja para crear y mantener nuevas formas de vida en común (Atkinson y Raboy, 1998). La radiotelevisión pública de Gran Bretaña desarrolló un modelo que ha sido imitado en varios países, especialmente en lo referente a sus mecanismos de financiación, que provienen de tres vías: la principal es el canon que aportan los ciudadanos "que supone alrededor del 75\% de su presupuesto. La segunda fuente la conforman sus actividades comerciales, que aportan un 20\% de los ingresos. Y, por último, las ayudas gubernamentales [...] que representan el 5\% de sus ingresos" (Ojer, 2009, p.138). Sin embargo, para el 2010, el gobierno británico anunció un cambio en la forma de financiación del Servicio Mundial de la BBC, como parte de un amplio programa de recortes presupuestarios (BBC, 2010).

En Suecia, aunque no existe un sistema de radiotelevisión público unificado por una sola institución, ha hecho carrera el concepto de 'Folkbildning' para describir el desarrollo de los medios de interés público. Con este concepto se designan dos intenciones: la primera describe la búsqueda de superación y conquista de conocimiento por los sectores desvalidos y la segunda se refiere a una visión paternalista con la que se busca transmitir "a cuenta gotas (...) la cultura de élite y [mejorar] el gusto de las masas" (Atkinson y Raboy, 1998, p.93). Es necesario resaltar que en este país los medios de interés público son financiados por los ciudadanos. Respecto a la función que deben cumplir, surgen posiciones encontradas: algunos consideran que la cultura y la educación deben ser la prioridad (perspectiva presente desde la legislación de 1925), otros consideran que el periodismo crítico es el elemento distintivo de estos medios y para otro sector de la población lo importante es la defensa de intereses locales y regionales en la época de la globalización.

En Bélgica, el servicio de radiodifusión y televisión pública ha estado la mayor parte del tiempo ligado a los dictámenes políticos del momento. Para la época de su creación, en 1930, este servicio no buscaba el funcionamiento democrático de los medios de comunicación, ni mucho menos permitir la expresión cultural y lingüística de las diferentes comunidades que habitan el territorio, sino "elaborar una estrategia de hegemonía [...] que permitiera controlar la información de carácter político" (Atkinson y Raboy, 1998,p.101). Para 1960 se promulgó una nueva ley que concedió “autonomía cultural al servicio y garantizó una política de información libre mediante la neutralidad obligatoria y una menor interferencia de las autoridades" (Atkinson y Raboy, 1998, p.102) paradójicamente, mientras se le concedía libertad de expresión al servicio, se restaban sistemáticamente los fondos para su financiación, juzgando su legitimidad en criterios de rentabilidad económica y ratings de audiencia. 
Según Ortiz Sobrino (2014) "lo más característico del modelo radiofónico español fue el nacimiento y desarrollo paralelo de la radio pública y la radio privada entre las décadas de los años 20 y 30 del pasado siglo y un reparto de papeles claramente marcado y sin apenas beligerancia empresarial" (p. 67). Sin embargo, esta 'convivencia' deriva en un sistema oligopólico, regido en la actualidad por 4 grupos empresariales: Radio Nacional de España, Prisa Radio, Atresmedia Radio (Onda Cero) y el Grupo COPE.

En Francia el mercado radiofónico está principalmente en manos de la iniciativa privada.

'El sector público agrupa [...] las emisiones internacionales de la radio pública francesa -Radio France Internacional-, las estaciones locales de radio en territorios de ultramar - como RFO-, cadenas públicas nacionales -France Info, France Inter, France Culture, France Musique y France Blue-, así como las redes locales o departamentales de radio y las redes de radio musicales y de servicios (FIP)' (Ortiz Sobrino, 2014, p.68).

Estos medios no incluyen publicidad dentro de su parrilla de programación, aunque emiten mensajes institucionales y de interés general.

Por su parte, Japón tiene uno de los sistemas más satisfactorios de radiotelevisión mixto, construido en torno a la piedra angular de un organismo de servicio público (NHK). ”El sistema de Japón combina la radiotelevisión de servicio público del estilo británico financiada mediante cánones, con la radiotelevisión comercial de tipo estadounidense que depende de los ingresos procedentes de la publicidad" (Atkinson y Raboy, 1998, p. 113). En este caso, a diferencia de otros países, la entidad de servicio público NHK posee mayor cantidad de trabajadores y aceptación por parte de las audiencias. Esto ha permitido que Japón tenga uno de los sistemas de medios públicos más competitivos a nivel mundial, que no permite la financiación del Estado, ni autoriza la propiedad única -monopolios- de distintos tipos de medios de comunicación.

En el contexto latinoamericano se encuentran referencias al caso de México, donde la radio de interés público surge como una manera de proyectar la cultura de élite, "atacando el gusto masivo y convocando a un gusto alejado del hábitus de clase de los grandes núcleos de audiencia” (Berlín, 2000, p.18). En el caso Argentino se encuentra que el servicio de radiodifusión ha sido promovido principalmente por el sector privado; "una de las [...] consecuencias de esta política fue la concentración de los principales sistemas de radiodifusión en los centros urbanos que interesaba a los anunciantes" (Gentino, 1995,p.138); sin embargo, se evidencia un cambio en cuanto al objetivo de los medios de interés público con la promulgación de la ley de medios Argentina (Ley 26.522 del 10 de octubre de 2009), en donde se reserva el 30\% del espectro a medios sin ánimo de lucro y se limita la cantidad de licencias que puede tener una misma persona. 


\section{Radiodifusión de interés público en Colombia}

Como ya mencionó, la resolución 0415 de 2010 define como emisoras de interés público la radio pública nacional de Colombia, las emisoras de la fuerza pública, emisoras territoriales, emisoras educativas, emisoras educativas universitarias y las emisoras para atención y prevención de desastres. Además de la normatividad mencionada existen algunas investigaciones patrocinadas por Ministerio de Tecnologías de la Información y las Comunicaciones, que dibujan un panorama del servicio de radiodifusión de interés público en Colombia. Uno de estos documentos es el Diagnóstico del Servicio de Radiodifusión de Interés Público (Gómez, 2003) que hace un estudio con 45 emisoras (7 emisoras de alcaldías y gobernaciones, 16 emisoras universitarias, 14 emisoras de la policía nacional, 8 emisoras del ejército nacional) y muestra aspectos propios de las estaciones de interés público en el país:

- Estructuras organizativas: resalta la presencia de profesionales en los grupos de trabajo que conforman las emisoras de interés público, pero echa de menos la inclusión de personal dedicado a la construcción de procesos a través de estrategias de comunicación; es decir, el personal que hace parte de la emisora se dedica principalmente a labores administrativas y de producción, más no al desarrollo de procesos comunicativos.

- Mecanismos de financiación: este ha sido un punto crítico a través de la historia de las emisoras de interés público en Colombia, pues la mayoría de las estaciones no cuenta con una asignación presupuestal permanente para su funcionamiento y consolidación; además, se encuentra una reclamación constante por parte de las emisoras a los lineamientos de financiación establecidos por la ley.

-Vínculos entre la comunidad y la emisora de interés público: al interior de las emisoras se manifiesta un interés por vincular las opiniones y sugerencias que los oyentes brindan a través de llamadas telefónicas sobre la programación; sin embargo, el diagnóstico evidencia que a estas opiniones se les resta valor cuando se enfrentan ante las propuestas del personal directivo y administrativo de la emisora. La programación no tiene en cuenta aspectos como el género, escolaridad y actividad laboral de los oyentes, pero es claro que ésta se orienta principalmente a satisfacer el gusto del público joven.

- Relación entre los objetivos institucionales y los fines de las emisoras de interés público: los objetivos de las emisoras de interés público se dispersan en infinidad de temas, sin que haya una claridad acerca de su utilidad. Varias de las instituciones con licencia para el manejo de emisoras de interés público no tienen claro para qué quieren la emisora, ni mucho menos, el valor agregado que ésta le brinda a la institución. 
'(...) las Emisoras de Interés Público deben fortalecer el diálogo y la interacción entre la sociedad civil y el Estado, desde el polo del Estado. Mediante el fortalecimiento de una cultura política madura y crítica, basada en mecanismos de información - oportuna, veraz y pertinente - y el estímulo y acompañamiento a formas de participación de los distintos sectores, las emisoras deben contribuir a la convivencia y a la ampliación de la democracia' (El' Gazi, 2008, p.9).

Otro aporte que realiza la Política de Radiodifusión Sonora de Interés Público (El'Gazi, 2008) es el establecimiento de principios que orientan este servicio. Entre éstos vale la pena destacar el aporte a la construcción de lo público, propiciando la comunicación entre el Estado y la ciudadanía y el aporte a la formación de ciudadanos competentes para la convivencia pacífica, la solidaridad y el respeto mutuos.

Para el año 2013, el Ministerio de Cultura convocó un encuentro de radios de interés público territorial, con el objetivo de motivar en estos medios la creación de espacios democráticos y de convivencia, de igual manera se abordaron temáticas como audiencias, políticas de programación, construcción de lo público y fortalecimiento ciudadano. Este encuentro, que contó con la participación de 12 emisoras, dio origen a la Red de Radio Pública Territorial de Colombia. Ese mismo año se realizó el segundo encuentro de la Red, que brindó asesoría para el manejo de medios digitales al servicio de la radio. El espacio permitió la planeación para el fomento de la creación de contenidos culturales de calidad, incluyentes y pertinentes (Ministerio de Cultura, 2013). A partir de la creación de esta Red, se comienza a dar respuesta a varias de las situaciones planteadas en los documentos y diagnósticos previos. El tercer encuentro fue en el año 2014 y contó con la participación del Centro de Memoria Histórica, que compartió varias herramientas para trabajar la memoria desde la radio de interés público. Desde aquí se vislumbra la gran responsabilidad que tienen este tipo de emisoras en la creación y fortalecimiento de una cultura de paz, dada la coyuntura social y política que vive el país en los últimos años. La Red de Radio Pública Territorial de Colombia, dio origen al programa 'Voces de Colombia' en donde mensualmente una de las estaciones miembro se encargaba de la producción y las otras emisoras aportaban en contenido para la emisión. Durante el 2014 y el 2015, Voces de Colombia fue una oportunidad de reflexión para la radio de interés público territorial respecto a su función como medio al servicio del Estado.

Es necesario mencionar que entre los encuentros y miembros de la Red de Radio Pública Territorial de Colombia no participan las emisoras universitarias. Según la entrevista realizada a Liliana del Pilar Ramírez (2016) Coordinadora del proyecto de fortalecimiento de radio comunitaria y de interés público de la Dirección de Comunicaciones del Ministerio de Cultura, esto se debe a la diferencia de intereses en el accionar, pues cada una responde a su institucionalidad: "Bajo los parámetros normativos tanto emisoras territoriales como universitarias hacen parte de la radio de interés público, sin embargo, se debe entender la naturaleza de estos medios y el 
tipo de organización que está a cargo de su gestión”. También se debe reconocer que las emisoras universitarias han trasegado un camino más largo respecto al trabajo asociativo, pues desde el año 2003 cuentan con la Red de Radios Universitarias (RRUC), que las agrupa en torno a experiencias similares, en búsqueda de opciones de financiación y el desarrollo de proyectos de comunicación (Basilago, 2009). Para el año 2015, la RRUC contaba con 56 universidades adscritas, estas pertenecen a '36 Instituciones de Educación Superior, de las cuales 34 emiten en F.M., 9 en A.M. y 13 lo hacen por Internet' (Ventín, 2015).

\section{Emisoras Universitarias de Interés Público}

Tras reconocer la ubicación que dentro de la legislación colombiana tienen las emisoras universitarias como servicios inscritos dentro de la radiodifusión de interés público, es necesario mencionar aspectos específicos de la radio universitaria. Aunque no es muy amplia la bibliografía al respecto, estos aportes establecen objetivos y funciones para la radio universitaria y abren múltiples caminos de estudio ${ }^{3}$.

Se pueden determinar varios usos de la radio en el contexto universitario y es necesario hacer claridad sobre ellos para comprender a qué 'radio universitaria' se hace referencia en este documento. Inicialmente, cabría realizar una diferencia entre el uso de la radio dentro de la universidad a nivel interno o local, como material pedagógico o producto de una clase y la radiodifusión universitaria que “(...) debe ser con carácter masivo y desde la propia universidad, por lo que no basta sólo utilizar al medio radiofónico, sino con producir y transmitir desde el recinto universitario (...)” (Perez, 2004, p.159)

También, es necesario distinguir entre la radio universitaria realizada y avalada por una determinada institución educativa de nivel superior y la radio hecha sólo por estudiantes universitarios que no es institucional (Perez, 2004) y que transmite haciendo uso de equipos de onda corta o a través de redes tecnológicas.

Para el caso específico de este documento, se hará alusión a la definición dada por Ventín (2015) sobre las radios universitarias al considerarlas medios de comunicación "que [tienen] un vínculo, ya sea en su estructura de producción, de organización o de contenidos, con una institución de educación superior, cuya función es la difusión de la cultura, la ciencia y la educación, así como contribuir al desarrollo formativo de estudiantes" (p. 90).

Se comentarán ahora algunas perspectivas y funciones que han sido encomendadas a la Radio Universitaria a lo largo del tiempo. Inicialmente, se puede anotar que la emisora universitaria desempeña un papel de extensión que abre las puertas de la universidad a los oyentes, esto es lo que se define como "Modelo comunitario clásico" (Berlín, 2002), donde la emisora extiende a toda la sociedad la cultura institucional en los mismos formatos usados en las aulas. En este caso, la legitimidad de la emisora no se da por el medio en sí o por el uso que de ella haga la audiencia, sino por la institución y su carácter de producción de conocimiento especializado. Otra de las perspectivas o funciones que se ha asignado a la radio universitaria es la de difundir la cultura de una 
ciudad, región o país; entendiendo el término cultura como las diferentes expresiones y modos de hacer de los individuos y colectividades. Para la radio universitaria esto significa dar paso a la expresión de artistas, intelectuales, estudiantes, docentes, investigadores y comunidades (Quevedo, 2004).

Otra función asignada a estas emisoras es la difusión del conocimiento y del quehacer universitario mediado con las necesidades propuestas por la audiencia. Por tanto, la emisora universitaria sirve para posicionar la oferta educativa en el mercado, para establecer un vínculo entre la comunidad universitaria y la población externa, trascendiendo las paredes del centro educativo (Ortiz \& Marquez, 2006). Según Dido y Barberis (2006) la radio universitaria "no es un medio para desarrollar cursos a distancia, sino un medio de comunicación, con perspectiva universitaria, que incluye en su programación variedad de formatos, géneros y contenidos para crear un ámbito radiofónico interactivo de intereses y valor para su universo de audiencia” (p.12). El puente que se genera entre la comunidad universitaria y la población externa obliga a la radio universitaria a transitar entre el espacio institucional y el masivo. "En esta función pasa a primer plano el sentido educativo y cultural de las emisoras, como una diferenciación del resto del sistema de medios de determinado espacio social" (Prieto citado por Ortiz \& Marquez, 2006).

\section{Emisoras Universitarias de Interés Público en Colombia}

El nacimiento de emisoras con un interés distinto al comercial se ubica con el surgimiento de la Radio Nacional de Colombia (1940) y la HJCK (1950). La radio educativa nace con el sistema de escuelas radiofónicas de Acción Cultural Popular, más conocida como radio Sutatenza en 1948 (Rincón, 1997). La primera emisora universitaria del país fue la 'Emisora Cultural de la Universidad de Antioquia' (1933), seguida por la fundación de 'Radio Bolivariana' de la Universidad Pontificia Bolivariana (1948). La mayoría de las emisoras universitarias en Colombia surgen como proyectos de individuos, que luego fueron acogidos por las universidades como una propuesta institucional (Gaviria, 2008).

El panorama de la radiodifusión de interés público universitario en Colombia es diverso y depende, en muchos casos, de la institución de educación superior a la cual pertenezca la emisora. Incluso a nivel administrativo no existe claridad sobre la dependencia en la que se suscribe: algunas emisoras hacen parte de departamentos de comunicación social o medios de información, otras dependen de instancias administrativas como rectoría, secretaría general, dirección de extensión universitaria o consejo superior, generando para cada caso mayor o menor cercanía con los lineamientos del poder central administrativo de la institución educativa.

La legislación, a través de la Resolución 0415 de 2010, establece que son emisoras educativas universitarias aquellas encargadas de (...) "la transmisión de programas de interés cultural, sin ninguna finalidad de lucro, con el objeto, entre otros, de difundir la cultura, la ciencia y la educación, de estimular el flujo de investigaciones y 
de información científica y tecnológica aplicada al desarrollo, de apoyar el proyecto educativo nacional y, servir de canal para la generación de una sociedad mejor informada y educada. Este servicio se prestará a través de instituciones de educación superior, legalmente reconocidas y acreditadas institucionalmente por el Ministerio de Educación Nacional".

En el Decreto 2805 de 2008 se clasificaba como Emisoras Universitarias de Interés Público sólo a las emisoras de universidades públicas; sin embargo, la actualización posterior de la legislación - Decreto 0415 de 2010 - otorga a todas las instituciones de educación superior reconocidas y acreditadas por el Ministerio de Educación, la posibilidad de prestar el servicio de radiodifusión de interés público educativo. Según Ventín (2015) entre las emisoras universitarias adscritas a la RRUC, el 55\% (31) cuentan con licencia de radio de interés público; un 22\% (12) funciona con licencia de radio comercial y un 23\% (13) no cuenta con licencia al ser emisoras que se trasmiten únicamente a través de internet.

En el documento Política del Servicio de Radiodifusión Sonora de Interés Público (El’Gazi, 2008), se mencionan labores de la radio universitaria aún hoy vigentes: difundir información acerca de la actividad investigativa y de formación propia de la institución, para que pueda ser aplicada al desarrollo social y económico; y soportar el proyecto educativo nacional y servir de canal para la formación de una sociedad mejor educada e informada. Este escrito plantea además que la radio universitaria es un espacio alternativo al comercial, que entrega información a aquellos sectores de la audiencia que no pueden acceder al conocimiento de punta: Las emisoras universitarias cuentan con herramientas de análisis de problemáticas locales y globales, que pueden aportar a las audiencias elementos para el conocimiento de la complejidad social.

Tomando como referencia puntos clave para el funcionamiento y desarrollo de una emisora - organización, fuentes de financiación, producción técnica, programación y audiencia- se presenta a continuación un panorama del sector, teniendo en cuenta los aportes de diferentes autores.

\section{Organización}

En términos de organización, la falta de identidad de las emisoras universitarias es señalada como una carencia: 'Algunas instituciones tienen emisora para darse imagen; otras porque quieren dar un aporte cultural; otras que quieren mejorar sus ventas y matriculas' (Rincón, 1997, p.1).

Un avance en la consolidación de un objetivo común para la radio universitaria se ve reflejado en la conformación en el año 2003 de la ya mencionada Red de Radio 


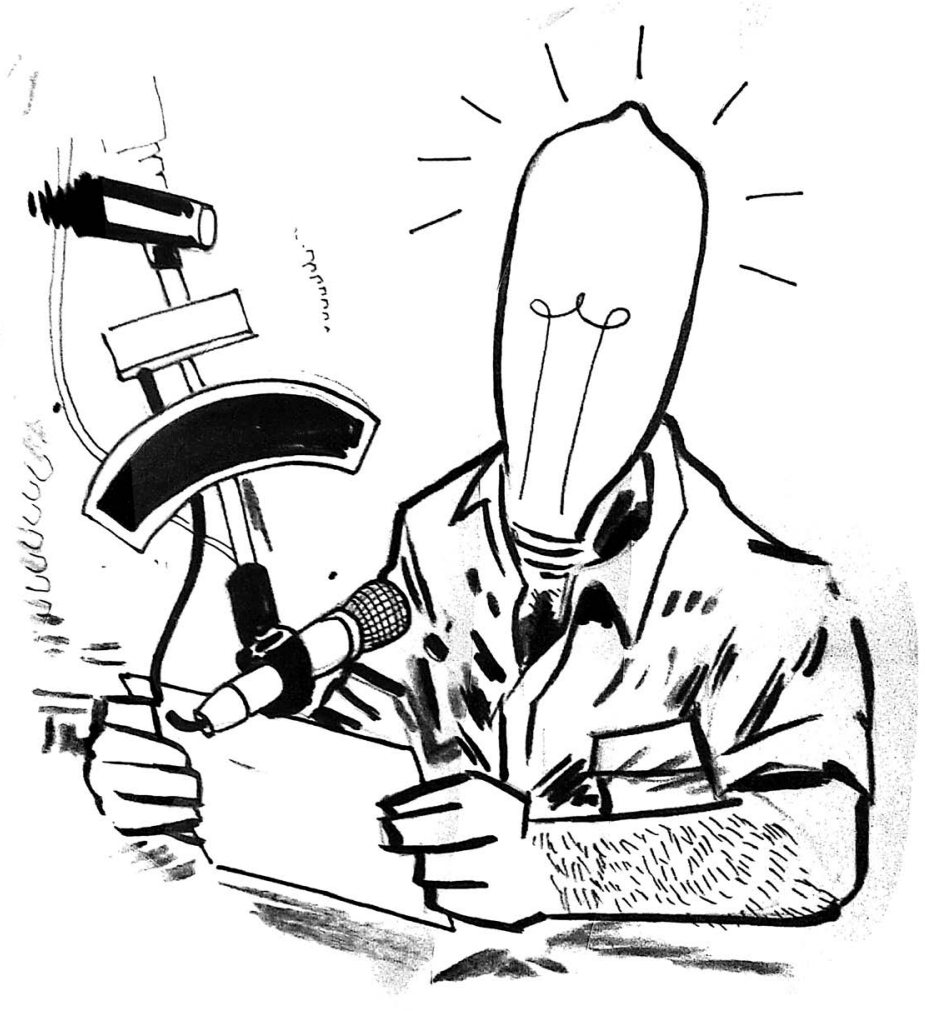

Universitaria de Colombia (RRUC). Sus primeras acciones estuvieron orientadas a lograr una mejor definición por parte del Ministerio de Comunicaciones en cuanto a las posibilidades de financiación de la radio universitaria y "un reconocimiento de su naturaleza y acción educativa con relación al pago de los derechos de autor" (Gaviria, 2008, p.6). La RRUC también se ha convertido en "un canal alternativo de distribución de contenidos de orden académico $\mathrm{y},(\ldots)$ especialmente, de alto impacto social y cultural" (Gaviria, 2008, p.6)

La tesis de maestría de la Universidad del Norte, Las Radios Universitarias en Colombia (Arnedo, 2009), tiene como objetivo investigar el estado de las Emisoras de Interés Público Universitario en el país, de acuerdo con la función que éstas deben cumplir según la normatividad. En este documento se menciona otro punto a favor de la organización de las emisoras universitarias, al resaltar que la mayoría cuenta con personas vinculadas laboralmente tiempo completo, lo que garantiza la gestión en forma permanente. Además, tienen conformados comités de evaluación, seguimiento y toma de decisiones.

Estos avances a nivel organizativo, han permitido que la misión y los objetivos de

142 las emisoras se alineen con "los propósitos consignados en el Plan de Radiodifusión Nacional, haciendo énfasis en la difusión de cultura, educación y civismo" (Arnedo, 2009) Sin olvidar las prioridades establecidas por cada emisora.

\section{Fuentes de financiación}

Cuando la ley establece que las Emisoras Universitarias de Interés Público no tendrán fines de lucro, se plantea el que ha sido uno de los problemas tradicionales de estas estaciones: la asignación de recursos a la radio educativa y cultural "no está directamente relacionada con variables de eficiencia comunicativa o cumplimiento de las metas anuales, sino [que] (...) depende de criterios políticos, unipersonales y de disponibilidad de fondos, afectando la credibilidad que pueda lograrse en torno de estos procesos de planeación y evaluación” (Berlín, 2000, p.13).

Los patrocinios y anuncios comerciales, que para las emisoras con otras estructuras de programación son el modo de equilibrar los presupuestos, transgreden los límites legales en el caso de la radiodifusión universitaria de interés público: "la legislación 
presenta dificultades que impiden la autofinanciación de las emisoras, al tiempo (...) que los aportes, auspicios o alianzas, a pesar de ser una gran ayuda, puedan convertirse en una amenaza, toda vez que los intereses de quienes contribuyen con la financiación (...) puedan ir en contravía de los de la emisora” (Arnedo, 2009, p.95)

\section{Producción técnica}

Para las emisoras universitarias de interés público, el reducido presupuesto económico dificulta la adquisición de equipos y tecnologías, impidiendo sostener la producción de programas por largo tiempo o garantizar la emisión las 24 horas del día. Esto es notorio en las parrillas que evidencian un elevado porcentaje de programación musical. Sin embargo, es necesario resaltar que "el manejo de la música, sus géneros, formas, contenidos, autores y presentaciones, se ha convertido en un propuesta innovadora de la radio universitaria a diferencia del esquema de un disco tras otro, tipo discoteca, que hemos acostumbrado a escuchar en las emisoras comerciales" (Arnedo, 2009, p.99).

En cuanto al personal, las emisoras "cuentan con equipos de trabajo conformados por profesionales de la Comunicación Social y el Periodismo, técnicos y tecnólogos en producción y transmisión; así como con el apoyo de colaboradores interdisciplinarios y expertos en diferentes áreas, además del apoyo de trabajadores en el área administrativa" (Arnedo, 2009, p.93), lo que permite plantear que, con los equipos técnicos y tecnologías adecuadas, se podría garantizar un mejor trabajo.

En este punto, se presenta una diferencia significativa respecto al balance presentado por el Ministerio de Comunicaciones (Gómez, 2003), pues como ya se expuso, en años anteriores los profesionales de la comunicación y el periodismo, no hacían parte de los equipos de producción de las emisoras de Interés Público. En el trabajo de Arnedo (2009), se evidencia cómo las Emisoras Universitarias han ampliado sus equipos de trabajo vinculando profesionales de diferentes áreas -incluida la Comunicación Social-, generando un impacto positivo en la producción y direccionamiento estratégico de las emisoras.

En cuanto a las tecnologías de emisión y producción, es necesario realizar un análisis sobre el uso de las tecnologías de información y comunicación (TIC) en la Radio Universitaria. Las TIC han venido modificando la programación, las narrativas, los lenguajes, los paisajes sonoros y los hábitos de las audiencias. Zambrano (2012) propone una radio universitaria donde los contenidos 
sean complementados con aplicaciones tecnológicas y lenguajes multimedia: "retomar los objetivos radiales de informar, orientar y entretener e incentivar la creatividad y experimentación a través de las redes sociales" (p..129). Ya son evidentes las diferencias entre las radios universitarias que aún reproducen modelos tradicionales frente a las que están afrontando los cambios e incorporando las tecnologías de la información y comunicación como parte de su producción técnica y de contenido.

\section{Programación}

Las temáticas tratadas en la programación de las emisoras universitarias de interés público en Colombia son bastante variadas. En las parrillas se crean franjas que cubren los espacios de lunes a viernes y estructuras mixtas para los fines de semana.

En la investigación de Ventín denominada" la radio universitaria en Colombia: concepción de sus estructuras de gestión, producción y programación para la creación de una tipología de dirección de medios" (2015), que incluye aquellas emisoras que funcionan con licencia de interés público, se menciona que:

"Los programas culturales se producen a partir de contenidos musicales y hablados. Los musicales se dan en espacios programáticos en los que se presentan obras en contexto (autor, año, género, idiosincrasia). Los géneros y estilos musicales que imperan son: folclore colombiano y músicas del mundo (con mucha presencia de estilos latinoamericanos), destinados a promocionar la identidad como nación a partir de la riqueza sonora regional" (Ventín, 2015, p.671).

Respecto a las franjas de entretenimiento Ventín (2015) señala cómo estas ocupan gran porcentaje de la programación de las emisoras y son, casi siempre, listas musicales que se insertan sin ninguna contextualización.

\section{Audiencia}

El conocimiento de las audiencias es una de las debilidades más notorias de las emisoras universitarias. El contacto que las emisoras establecen con su público se limita a las llamadas, reportes de sintonía, chat y correos electrónicos. Las emisoras no cuentan con un mecanismo o modelo de evaluación o seguimiento de la audiencia (Arnedo, 2009). Existe una aproximación de tipo cuantitativo, que toma como referencia los mecanismos nacionales de estudio de medios (EGM). De igual forma se debe reconocer la construcción de audiencias que se da desde lo virtual. Es muy común observar cómo las emisoras cuentan con Fan Page, chats, cuentas en twitter y otras redes sociales que les permiten generar un contacto permanente con ciertos grupos de audiencias. Sin embargo, es necesario llamar la atención sobre la manera como las Emisoras Universitarias están construyendo su público, pues lo hacen de 
forma similar a las radios de tipo comercial, centrándose sólo en conocer las cifras de quienes potencial o realmente las sintonizan. Al respecto, es necesario referenciar la tesis de maestría de la Universidad del Norte, 'Aportes para la caracterización de las audiencias de las Emisoras Universitarias de Interés Público en Colombia' (Díaz, 2012) en la que se propone una metodología cualitativa, a partir de la teoría de las mediaciones múltiples de Guillermo Orozco Gómez (2006), para conocer y describir quienes son las audiencias de dos emisoras universitarias del país: Univalle Estéreo ${ }^{4}$ de la Universidad del Valle y UdeC radio ${ }^{5}$ de la Universidad de Cartagena. Esta investigación devela la importancia de que las emisoras conozcan y trabajen en función de satisfacer las demandas de sus oyentes, pues sólo así tendrán parámetros claros que guíen su parrilla de programación y permitan reorientar estrategias que favorezcan su sostenibilidad, sin detrimento de los estatutos legales que la rigen. Las Emisoras Universitarias de Interés Público constituyen para las audiencias un espacio alterno para el disfrute y entretenimiento, pero es poco perceptible la capacidad que estos medios tienen de generar opinión y difundir el conocimiento que se produce desde la academia.

Esta revisión de literatura sobre las Emisoras Universitarias de interés público en Colombia, muestra un panorama con varios puntos sobre los que es necesario trabajar y profundizar para garantizar la sostenibilidad y el cumplimiento de los objetivos trazados para estos medios. También, es necesario indicar que el quehacer supera en muchos casos a la reglamentación. Los parámetros establecidos en la normatividad, permiten que las radios universitarias de interés público trabajen de formas diferentes. Por tanto, es necesario continuar avanzando en la reflexión sobre el papel de la radio universitaria en la vida contemporánea y su relación, más allá de la legislación, con los medios de interés público. Una tarea pendiente es el análisis del rol de la radio universitaria en el postconflicto. Desde esta perspectiva la participación de la comunidad académica no puede continuar siendo puntual en la generación de comentarios de acuerdo a un tema o especialidad, es necesario trascender la función informativa y realizar una labor pedagógica, que consolide lazos entre la universidad y la comunidad.

\section{Notas}

${ }^{1}$ Comunicadora Social- Periodista. Especialista en Comunicación Organizacional. Magister en Comunicación.

${ }^{2}$ Gran parte de la información aquí incluida fue compilada para el desarrollo de la tesis de Maestría en Comunicación de la Universidad del Norte: 'Aportes para la caracterización de las audiencias de las Emisoras Universitarias de Interés Público en Colombia, desde el modelo de las mediaciones múltiples, para contrastar los usos y expectativas de las audiencias con las funciones que le asigna la ley a estos medios' (Díaz, 2012). Posterior a la entrega, sustentación y aprobación de la tesis, se realiza una actualización de fuentes, datos y avances para la elaboración del presente artículo de revisión. Nota de la autora.

${ }^{3}$ Algunos aportes sobre la importancia estratégica de las Emisoras Universitarias dentro del ámbito de la radiodifusión de interés público se mencionan en la ponencia: “Aportes para la caracterización de las audiencias de las Emisoras Universitarias de Interés Público en Colombia, desde el modelo de las mediaciones múltiples, para contrastar los usos y expectativas de las audiencias con las funciones que le asigna la ley a estos medios, presentada por Díaz, A. \& Vega, J. (2011) en el primer encuentro de la Red de Radios Universitarias de Latinoamérica y el Caribe. 
${ }^{4}$ Univalle Estéreo: http: / / emisora.univalle.edu.co/

${ }^{5}$ UdeC radio: http://udecradio.unicartagena.edu.co/

\section{Referencias}

Arnedo, B. (2009) La radios universitarias en Colombia. Tesis de Maestría. Universidad del Norte. Barranquilla, Colombia.

Atkinson, D. y Raboy, M. (1998) La radio y la T.V. de servicio público: el desafío del siglo XXI. Revista estudios y documentos de comunicación de masas No. 111. Unesco, Paris, Francia.

Basilago, J. (2009) Red de Radio Universitaria de Colombia. RadioWorld.com Tomado de: http://www. nxtbook.com/nxtbooks/newbay/rwla_200912/index.php?startid=10

BBC Mundo (2010). Cambio en la financiación del Servicio Mundial de la BBC. 20 de octubre de 2010. Tomado de: http://www.bbc.com/mundo/noticias/2010/10/101019_reino_unido_bbc_ reforma_licencia_fp

Berlin, I. (2000). El derecho a decir: radios universitarias y educativas en México. Revista latina de comunicación social 27, 43. Marzo. Tenerife, Canarias; España

Decreto 2085 de 2008 reglamento de servicio de radiodifusión sonora 2008. Tomado de http: / www. radiouniversitaria.org/

Díaz, A. (2012) Aportes para la caracterización de las audiencias de las Emisoras Universitarias de Interés Público en Colombia, desde el modelo de las mediaciones múltiples, para contrastar los usos y expectativas de las audiencias con las funciones que le asigna la ley a estos medios. (Tesis de Maestría). Universidad del Norte. Barranquilla, Colombia.

Díaz, A. \& Vega, J. (2011). Aportes para la caracterización de las audiencias de las Emisoras Universitarias de Interés Público en Colombia, desde el modelo de las mediaciones múltiples, para contrastar los usos y expectativas de las audiencias con las funciones que le asigna la ley a estos medios. (Ponencia) Primer encuentro de la Red de Radios Universitarias de Latinoamérica y el Caribe. Ciudad de México, México. Disponible en: https://sites.google.com/a/rrulac.org/rrulac/ encuentro-rrulac-2011/ponencias

Dido, J. \& Barberis, S. (2006) Radio Universitarias. Principios, funciones, objetivos. Universidad Nacional de La Matanza. Buenos Aires, Argentina.

El'Gazi, J. (2008) Política de radiodifusión sonora de interés público: radio y ampliación de lo público. Ministerio de comunicaciones. Bogotá, Colombia.

Gaviria, G. (2008). La radio universitaria en Colombia. Tomado de http: / /www.radiouniversitaria.org/ Gentino, O. (1995). Las industrias culturales en la Argentina. Dimensión económica y políticas públicas. Ediciones Colihue. Buenos Aires, Argentina.

Gomez, G. y Quintero, J. (2003) Diagnóstico del servicio de radiodifusión de interés público. Dirección general de comunicación social, Ministerio de comunicaciones. Bogotá, Colombia.

Ojer, T. (2009) El modelo de financiación de la BBC. Comunicación y Sociedad. Vol. XXII, Núm. 1. 2009. P. 137-160 
Orozco, G. (2006) Los estudios de recepción: de un modo de investigar a una moda, y de ahí a muchos modos. En Saintout, F \& Ferrante, N. (Comp.) ¿y la Recepción? balance crítico de los estudios sobre el público. La Crujía ediciones, Buenos Aires, Argentina.

Ortiz, A., \& Marquez, M. (2006). El sistema universitario de radio. In UABC (Ed.), Los medios de comunicación en baja california (pp. 10).

Ortiz Sobrino, M., Rodriguez, D. \& Jaques, J. (2014) La radio universitaria en el siglo XXI: perspectiva hispano-francesa. Revista de educación mediática y Tic, Edmetic. 3 (1) pp. 61 -86

Perez, L. (2004). La radiodifusión universitaria y los circuitos cerrados de radio en las instituciones de educación superior: una propuesta para la expresión de los estudiantes. Revista mexicana de ciencias políticas y sociales, 192, 33. Septiembre - diciembre. Universidad nacional Autónoma de México

Quevedo, L. D. (2004). La radiodifusión universitaria a la búsqueda de sentido. Reencuentro análisis de problemas universitarios. 039, 8. Abril. Universidad Autónoma Metropolitana, Xochimilco México.

Resolución 0415 de 2010. Estructura de propiedad de la radio en Colombia. Tomado de http: / /www. mintic.gov.co/portal/604/w3-article-3797.html

Rincón, O. (1997) La radio en la universidad javeriana. Tomado de http: / / www.radio.uady.mx/ ariadna/articulos/javeriana.html

Ventín, J. (2015) La radio universitaria en Colombia: concepción de sus estructuras de gestión, producción y programación para la creación de una tipología de dirección de medios. (Tesis doctoral) Universidad Complutense de Madrid, Facultad de Ciencias de la Información, Departamento de Periodismo. España.

Zambrano, W. (2012). Radiografía de las emisoras universitarias de Colombia. Folios No. 28. Facultad de Comunicaciones. Universidad de Antioquia, Medellín. Colombia.

Recibido: abril 2016/ Aprobado: mayo 2016 